\title{
Proporcionalidade: um olhar a partir da TAD Proportionality: a look from ATD
}

\author{
MARIA SÔNIA LEITÃO MELO VIEIRA ${ }^{1}$ \\ MARCELO CÂMARA DOS SANTOS ${ }^{2}$
}

\begin{abstract}
Resumo:
Este estudo tem por objetivo identificar em textos acadêmicos as razões de ser e a ecologia didática da proporcionalidade. Trata-se aqui, de um recorte de uma pesquisa em andamento no âmbito do Curso de Doutorado. Tomamos como aporte teórico/metodológico elementos da $T A D$, relacionados a ecologia didática de um saber. Partimos do questionamento: como se dá a ecologia deste saber na literatura acadêmica? Os resultados preliminares apontaram que as razões de ser, deste saber encontra-se no desenvolvimento do raciocínio proporcional principalmente junto ao ensino dos saberes razão, proporção e função linear. A perspectiva didática mais apontada é a resolução de problemas em contextos variado fazendo articulação com o estudo dos números racionais. A proporcionalidade, no ecossistema Matemática da Educação Básica, habita principalmente as subáreas Álgebra, Geometria e Números. E tem como nicho ecológico: razão, proporção, semelhança e função linear.
\end{abstract}

Palavras-chave: TAD. Ecologia didática. Razões de ser. Proporcionalidade.

\section{Resumen:}

Este estudio tiene por objetivo identificar en textos académicos las razones de ser y la ecología didáctica de la proporcionalidad. Se trata aquí, de un recorte de una investigación en curso en el ámbito del Curso de Doctorado. Tomamos como aporte teórico / metodológicos elementos de la TAD, relacionados con la ecología didáctica de un saber. Partimos del cuestionamiento: ¿cómo se da la ecología de este saber en la literatura académica? Los resultados preliminares apuntaron que las razones de ser, de este saber se encuentra en el desarrollo del raciocinio proporcional principalmente junto a la enseñanza de los saberes razón, proporción y función lineal. La perspectiva didáctica más apuntada es la resolución de problemas en contextos variados haciendo articulación con el estudio de los números racionales. La proporcionalidad, en el ecosistema Matemática de la Educación Básica, habita principalmente las subáreas Álgebra, Geometría y Números. Y tiene como nicho ecológico: razón, proporción, semejanza y función lineal.

Palabras clave: TAD. Ecología didáctica. Razones de ser. Proporcionalidad.

\section{Résumé :}

Cette étude vise à identifier dans des textes académiques les raisons d'être et l'écologie didactique de la proportionnalité. Ceci est une revue d'une recherche en cours dans le cadre du cours de doctorat. Nous prenons comme éléments de contribution théoriques / méthodologiques du TAD, liés à l'écologie

1 Universidade Federal de Pernambuco, Brasil, soniamatematic25@gmail.com, Registro público é http://orcid.org/0000-0002-4661-8207

${ }^{2}$ Universidade Federal de Pernambuco, Brasil, marcelocamaraufpe@ yahoo.com.br, http://orcid.org/0000-00033707-4807 
didactique d'un savoir. Nous partons du questionnement : comment l'écologie de ces connaissances se situe-t-elle dans la littérature académique? Les résultats préliminaires ont montré que cette connaissance s'expliquait par le développement du raisonnement proportionnel principalement par l'enseignement de la raison, des proportions et de la fonction linéaire. La perspective didactique la plus pointée est la résolution de problèmes dans des contextes variés en articulant avec l'étude des nombres rationnels. La proportionnalité, dans l'écosystème mathématique de l'éducation de base, habite principalement les sous-zones Algèbre, Géométrie et Nombres. Et il a comme niche écologique : raison, proportion, similitude et fonction linéaire.

Mots-clés : TAD. Écologie didactique. Raisons d'être. Proportionnalité.

\section{Summary}

This study aims to identify in academic texts the reasons for being and didactic ecology of proportionality. This is a review of an ongoing research within the PhD course. We take as a theoretical / methodological contribution elements of the TAD, related to didactic ecology of a knowledge. We start from the questioning: how does the ecology of this knowledge take place in the academic literature? The preliminary results pointed out that the reasons for this knowledge lie in the development of proportional reasoning mainly with the teaching of reason, proportion and linear function. The most pointed didactic perspective is the problem solving in varied contexts making articulation with the study of rational numbers. Proportionality, in the Mathematical ecosystem of Basic Education, inhabits mainly the subareas Algebra, Geometry and Numbers. And it has as ecological niche: reason, proportion, similarity and linear function.

Keywords: TAD. Didactic ecology. Reasons to be. Proportionality. 


\section{Introdução}

O estudo que tratamos aqui, faz parte de uma pesquisa em andamento, em Didática da Matemática, no âmbito do Doutorado em Educação Matemática e Tecnologia da UFPE, na qual temos por objetivo investigar a ecologia do objeto de saber proporcionalidade no Ensino Fundamental sob a ótica da TAD. Neste estudo nosso objetivo é identificar as razões de ser na ecologia didática da proporcionalidade, localizando habitats e nichos, na análise de textos acadêmicos - artigos em periódicos.

Pretendemos trazer luz ao debate sobre o saber proporcionalidade. Partindo do questionamento: como a literatura especializada, artigos em periódicos, tratam esse saber? Para tanto, realizamos nossa revisão de literatura, tomando a base de dados Periódicos da CAPES, como base principal deste ponto de nossa pesquisa.

Nossa metodologia de pesquisa partiu da análise de nove artigos acadêmicos. As categorias, de análise dos textos acadêmicos, surgiram a partir da adaptação de alguns elementos propostos por Chaachoua (2017), para a análise de livros didáticos tais como: o momento da publicação (com exceção de testos clássicos como Post, Behr e Lesh (1995), Spinillo (2002) e Bongiovanni (2005), os demais têm em média de 10 anos de publicação; a representatividade da obra (em periódicos (A1 e B2), a partir da classificação da CAPES); e análise ecológica dos textos sobre as condições modificáveis e não modificáveis do saber proporcionalidade nas instituições.

Nosso aporte teórico se situa na Teoria antropológica do Didático - TAD, que emerge dos estudos do pesquisador Yves Chevallard, desde a década de 90, do século XX e, muito estudada atualmente na Educação Matemática, principalmente no seio da Didática da Matemática.

\section{Nosso primeiro olhar}

Em nossa busca, no banco de dados dos periódicos da CAPES, selecionamos os textos em que os termos - proporcionalidade, proporção ou raciocínio proporcional estivessem presentes no título. Observamos que o termo proporção, surge relacionado a Estatística, fazendo parte dos saberes a serem estudados por esse campo de conhecimento, como por exemplo no texto de Fonseca (2018), que trata da "variação-proporção: um novo formato para apresentação de dados".

A palavra proporção quando pesquisada, nos títulos de artigos de diferentes áreas, apresenta, em nossa análise, diferentes ideias: a ideia de razão, ideia de proporção e a ideia de porcentagem. 
Observamos que o "termo proporção áurea" é muito utilizado, em textos de diferentes áreas, para se referir a questões de estéticas, principalmente relacionado a medicina e a odontologia. Podemos observar como, em diferentes áreas de conhecimento, proporcionalidade se faz presente confirmando a importância de seu estudo para diferentes gerações na vida cotidiana.

\section{Proporcionalidade em nossa vida}

O conhecimento matemático surge na humanidade a partir da necessidade de se resolver problemas do cotidiano e, aos poucos, junto com seus registros, passa a ser conhecimento de muitos, de várias pessoas, de comunidades. Essa ampliação, do universo deste conhecimento, o eleva a categoria de um objeto de saber $^{3}$ social.

Embora a matemática esteja presente em nossa vida, a construção do conhecimento matemático é diferente da construção do conhecimento cotidiano, do conhecimento que ocorre a partir dos fenômenos da natureza. Os objetos matemáticos são construções abstratas de conhecimento realizadas por cada indivíduo. Só a representação desses objetos matemáticos é perceptível aos nossos olhos. São construções realizadas pela mente humana e associada a uma representação. Diariamente nos deparamos com situações do cotidiano que demandam a utilização de objetos matemáticos. Vamos tomar como o exemplo, o problema a seguir que denominamos de Problema da gelatina.

Quadro 1 -Problema de gelatina

\begin{tabular}{|c|c|c|}
\hline & & Problema da Gelatina \\
\hline $\begin{array}{l}\text { Dissolva uma porção de } \\
\text { mais } 250 \mathrm{ml} \text { de água fria ou gelada } \\
\text { rende aproximadamente } 5 \text { poções } \\
\text { quanto de gelatina em pó é necessá }\end{array}$ & ala & $\begin{array}{l}\text { a em pó, em } 250 \mathrm{ml} \text { ( } 1 / 4 \text { de litro) de água fervente. Adicione } \\
\text { taças. Leve à geladeira até adquirir consistência. Esta receita } \\
\text { a. Para preparar aproximadamente } 40 \text { porções de } 100 \mathrm{ml} \text { cada, }\end{array}$ \\
\hline $\begin{array}{l}5 \text { porções } \rightarrow 30 \mathrm{~g} \\
1 \text { porção } \rightarrow 30: 5=6 \mathrm{~g} \\
40 \text { porções } \rightarrow 40.6 \mathrm{~g}=\end{array}$ & 1 & $\begin{array}{l}\text { Logo se } 1 \text { porção de } 30 \mathrm{~g} \text { de gelatina em pó rende } \\
\text { aproximadamente } 5 \text { poções de } 100 \mathrm{ml} \text {, cada. Então para obter } \\
40 \text { poções de } 100 \mathrm{ml} \text { cada, preciso descobrir quantas vezes } \\
\text { terei que multiplicar as } 5 \text { porções para obter } 40 \text {. }\end{array}$ \\
\hline $\begin{array}{l}\text { ou, } \\
5 x=40 \\
x=8 \text { pacotes }\end{array}$ & 2 & \\
\hline
\end{tabular}

Fonte: produção dos autores

Embora, o preparo da gelatina, seja uma tarefa relativamente fácil de ser realizada no dia a dia, o conhecimento matemático que está em torno desta atividade requer um pouco mais de atenção

${ }^{3}$ Tomando como base a Teoria Antropológica do Didático. 
com relação a seu estudo. Trata-se de realizar operações com grandezas, envolve números racionais, a relação entre a quantidade de gelatina em pó e a quantidade de água, entre outros conhecimentos.

No entanto, podemos recorrer a alguns domínios para resolver tal tarefa. No domínio da Aritmética, por exemplo, basta observar que 40 é um múltiplo de 5, então 40 é divisível por 5 que resulta em 8, mas, "8 o que"? Gramas ou pacotes? Observando os cálculos realizados notamos que a resolução 1, apelou para o método de encontrar o valor da unidade. Já, a resolução 2, recorreu à álgebra, resolvendo uma equação do primeiro grau. Mas, poderia, também, encontrar o resultado sem a realização de cálculos escrito, por ser um problema relativamente simples, bastaria pensar quantas vezes teremos que multiplicar as 5 porções para obter 40, ou seja, encontrar o fator multiplicativo. Mas, a simples indicação de um número não seria suficiente para apresentar a resposta adequada. Pois, se respondêssemos com relação a quantidade de pacotes de gelatina seriam 8, mas, com relação a quantidade de gramas de gelatina seriam 240. Nos questionamos: será que essa solução, poderia ser encontrada por alunos dos anos iniciais, do ensino fundamental?

A proporcionalidade é um dos saberes matemáticos que faz parte das atividades mais básicas do nosso dia a dia, muito presente nas práticas sociais. Por exemplo, quando uma costureira amplia ou reduz um molde de uma roupa para adequá-la a determinado manequim; no momento do preparo da mistura da tinta, pelo pintor, adequando a quantidade de solvente proporcionalmente a tinta concentrada; quando calculamos a quantidade de medicamento a ser ministrado a uma criança, em razão do "peso" que ela possui; na leitura de uma planta baixa; no cálculo das parcelas nas compras a prazo; na interpretação das contas de água, de luz ou das faixas para recolhimento do imposto de renda; quando adequamos uma determinada receita culinária em razão do quantitativo de pessoas que serão servidas; no preparo de uma bebida quanto a quantidade de açúcar com relação à quantidade de líquido (suco de frutas, água, por exemplo.); entre outros.

Desde o químico ao cozinheiro, do engenheiro ao mestre-de-obras, o raciocínio proporcional passa a ser uma competência básica para a realização de sua profissão. Várias outras áreas profissionais, nas quais a proporcionalidade é indispensável para o desenvolvimento de suas atividades, ainda podem ser citadas, tais, como: arquitetura e urbanismo, topografia, tecnologia, enfermagem, design de modas, etc.

Comin (2000), destaca que a proporcionalidade, “é um instrumento universal de comparação, 
ela descreve uma relação de dependência entre grandezas com as razões ${ }^{4}$. Ela se faz presente em muitas situações cotidianas tanto no dia a dia das pessoas como objeto de estudo na escola, mas ainda é um objeto gerador de dificuldades tanto do ponto de vista da aprendizagem quanto do ensino. A esse respeito Costa e Allevato (2015, p.03) afirmam que

\begin{abstract}
Apesar de terem contato quase que diariamente com situações de proporcionalidade, os alunos tendem a apresentar algumas dificuldades em compreender o conceito; ajudá-los a desenvolver o raciocínio proporcional tem sido um grande desafio no período escolar, sendo essencial ao aprendizado de diversas disciplinas do Ensino Fundamental, Médio e Superior.
\end{abstract}

No nosso dia a dia lidamos com proporcionalidade em várias situações nas quais precisamos comparar duas grandezas, às vezes, sem nem se dar conta que estamos fazendo matemática. No preparo de alimentos aumentando ou diminuindo uma receita, ao dosar uma determinada medicação para uma criança quando a quantidade de gotas fica em função do peso, na análise de uma planta ou maquete, entre outros.

Proporcionalidade também faz parte da rotina de diferentes profissões como por exemplo na Agronomia - cálculo do espaço a ser cultivado por diferentes tipos de lavoura, na Arquitetura - elaboração de projetos compatíveis com os diferentes ambientes, no Direito - calculo proporcional de bens nas partilhas de heranças, na Engenharia das diferentes áreas - elaboração de diferentes tipos de plantas e protótipos, entre outras. Nas disciplinas escolares proporcionalidade está presente, além da Matemática, no estudo da Física, da Química, da Biologia e da Geografia, por exemplo.

Alguns estudos (TINOCO, 1996; SCHLIEMANN; CARRAHER, 1997; COSTA E ALLEVATO, 2015) discutem que o trabalho de proporcionalidade nas escolas se restringe quase que exclusivamente à utilização da regra de três, baseando-se apenas nas propriedades de razões equivalentes, ou seja, dadas duas razões equivalentes $\frac{a}{b}=\frac{c}{d}$, se as igualdades $\frac{a}{b}=\frac{c}{d} \mathrm{e}$ $a \cdot x=b \cdot c$ são verdadeiras, portanto, $x=b \cdot \frac{c}{a}$.

Proporcionalidade é um conceito matemático muito estudado por vários pesquisadores. Para Vergnaud (1988) proporcionalidade é uma relação multiplicativa entre as quantidades de dois espaços-medidas. Van de Walle (2009) apresenta proporcionalidade como a igualdade entre duas relações. De acordo com Lima et al (2010), se $\mathrm{f}: \mathrm{R}+\rightarrow \mathrm{R}+$ é uma proporcionalidade, então, $x 1, x 2$ do $f(x 1)=y 1, f(x 2)=y 2$, tem-se y1/x2=y2/x2. De acordo com Costa Júnior (2010) resolver problemas que envolvem o conceito de proporcionalidade é muito mais que

\footnotetext{
${ }^{4}$ Elle est um instrument universel de comparaison, elle décrit une relation de dépendance entre grandeurs com as razões. (COMIN, 200, P.05).
} 
aplicar algoritmos, como a regra de três, por exemplo, normalmente associada à proporcionalidade. Para Costa e Allevato (2015) proporcionalidade é a relação entre duas variáveis (grandezas) proporcionais, porém os autores alertam que

\begin{abstract}
se o professor, em seu trabalho com o conteúdo, utiliza apenas esse tipo de estratégia está deixando de explorar as relações existentes entre as grandezas e, com isso, os alunos perdem a oportunidade de desenvolver o raciocínio proporcional. É recomendável identificar as características, isto é, as relações numéricas apresentadas em cada situação, assim como o fator invariante que permite exprimir a relação matematicamente. Dessa forma, entendemos que em problemas envolvendo o conceito de proporcionalidade devemos explorar as relações existentes entre as grandezas consideradas, bem como enfatizar a relação desse conceito com outros conceitos matemáticos.
\end{abstract}

Os autores ainda destacam que "a proporcionalidade não é apenas um conteúdo matemático, mas um 'formador' de estruturas cognitivas para a compreensão de outros importantes conceitos matemáticos, tanto nas questões numéricas, como naquelas que envolvem Medidas e Geometria” (COSTA E ALLEVATO, 2015, p.03).

Mas que o que vem a ser mesmo proporcionalidade, são apenas regras para se operar com proporção? E o raciocínio proporcional o que vem a ser? Então o saber é a proporção e a razão seria um nicho desse saber? Qual seu papel nessa ecologia? Qual a razão de ser desses "termos" na literatura especializada?

Entendemos que neste momento não será possível apresentar respostas para estes questionamentos, entretanto, nossos estudos em diversos artigos acadêmicos, estão nos permitindo localizar, na ecologia didática, algumas razões de ser, bem como, o(s) habitat(s) e nichos para o objeto de saber proporcionalidade.

\title{
Um pouco sobre a Teoria Antropológica do Didático - TAD
}

$\mathrm{Na} \mathrm{TAD}$, a problemática ecológica nos ajuda a questionar sobre as condições de vida dos objetos de saber, no seio das instituições. No nosso caso, a ecologia se refere as condições e restrições para a vida de um saber em um determinado ecossistema. A ecologia didática tem sua origem nos estudos de Yves Chevallard (1994). A atividade matemática e, por consequência, a atividade de estudo da matemática se situa no conjunto das atividades humanas, que para a TAD pode ser analisada a partir do estudo das suas organizações matemáticas e didáticas.

As condições modificáveis e não modificáveis por que passa o saber proporcionalidade, no interior do saber a ser ensinado, ou seja, no estudo das relações entre o saber proporcionalidade e o seu meio, nos remete a problemática ecológica. A problemática ecológica está na maneira 
de se questionar e de se buscar respostas para a razão de ser de determinados objetos: "De onde vem esses novos objetos de ensino? Como eles chegam até aqui? Quais as interpelações que existem com outros objetos? E, também, sobretudo: porque eles chegam até lá? ... sem me interrogar não somente o que é [...] mas ainda me pergunto por que isso, não é? (CHEVALLARD, 1994, p .05).

Neste estudo, partiremos da ecologia didática para buscar respostas aos questionamentos: qual as razões de ser do saber proporcionalidade na literatura acadêmica? Onde habita este saber e quais nichos possui? Levamos em consideração a noção de ecossistema, habitat e nicho ecológico para a realização, da análise da ecologia ${ }^{5}$ do saber proporcionalidade. Tomaremos como ecossistema ${ }^{6}$ a Matemática da Educação Básica, como habitat as subáreas da Matemática: Álgebra, Estatística e probabilidade, Grandezas e medidas, Geometria e Números.

\section{Razões de ser do saber proporcionalidade nos artigos acadêmicos}

Nos estudos de Ávila (1986, p. 03), identificamos um modelo epistemológico dominante para a proporcionalidade $-\operatorname{MRDP}_{(1)}($ se $\mathrm{y}=\mathrm{kx}$ ou $\mathrm{y} / \mathrm{x}=\mathrm{k}$; se $\mathrm{y}=\mathrm{k} / \mathrm{x}$ ou $\mathrm{xy}=\mathrm{k})$, cujo nicho ecológico se encontra na Álgebra, nas Grandezas e na Estatística. A nossa referência o domínio da Álgebra, se dá quando o autor se refere a "lei" de proporcionalidade, fazendo menção ao estudo da "função linear", que é um tema pertencente ao setor “função". Do mesmo modo, a alusão ao domínio das Grandezas e/ou da Estatística, quando ele se refere ao tema "grandezas ou varáveis diretamente proporcionais e as grandezas ou variáveis inversamente".

Definição 1. Diz-se que duas variáveis (ou grandezas) x e y são proporcionais - mais especificamente diretamente proporcionais - se estiverem assim relacionadas: $\mathrm{y}=\mathrm{kx}$ ou $\mathrm{y} / \mathrm{x}=\mathrm{k}$, onde $\mathrm{k}$ é uma constante positiva, chamada constante de proporcionalidade.

Definição 2. Diz-se que as variáveis x e y são inversamente proporcionais se $\mathrm{y}=\mathrm{k} / \mathrm{x}$ ou $\mathrm{xy}=\mathrm{k}$, onde $\mathrm{k}$ é uma constante positiva (constante de proporcionalidade).

Encontramos, também, em Post, Behr e Lehs (1995) a representação algébrica para proporcionalidade remetendo também a função linear y $=m x, \operatorname{MEDP}_{(2)}$.

A proporcionalidade é um exemplo simples, mas importante, de função matemática e pode ser representada como uma equação linear. Como tal, é uma ponte adequada e talvez necessária entre a experiência e modelos comuns e as representações mais abstratas que se expressarão de forma algébrica. A representação algébrica da

\footnotetext{
${ }^{5}$ A análise ecológica de um objeto de saber é organizada em torno de duas noções: o habitat que designa os lugares da vida e o ambiente conceitual deste objeto de saber e o nicho que designa a função desse objeto no sistema de objetos com os quais interage. (CHAACHOUA, 2017, P.05. Tradução nossa).

${ }^{6}$ Entendemos ecossistema como sendo o local onde se desenvolve um determinado sistema que possui uma ecologia própria, no caso em estudo, o sistema didático. (SANTOS E MENEZES, 2015, P.649).
} 
proporcionalidade $(\mathrm{y}=\mathrm{mx})$ abrange uma classe incrivelmente ampla de ocorrências físicas.

Lima (1997, p.92), já observava que "a função linear, dada pela fórmula $\mathrm{f}(\mathrm{x})=\mathrm{ax}$, é o modelo matemático para os problemas de proporcionalidade". E, nós, tomamos como $\operatorname{MEDP}_{(3)}$. O autor ainda, acrescenta que "a proporcionalidade é, provavelmente, a noção matemática mais difundida na cultura de todos os povos e seu uso universal data de milênios". Ele justifica seu argumento, apresentado uma citação do livro Aritmética Progressiva, de Antônio Trajano, adotado no Brasil até a década de 1960, livro que chegou a ter mais de oitenta publicadas.

Diz-se que duas grandezas são proporcionais quando elas se correspondem de tal modo que, multiplicando-se uma quantidade de uma delas por um número, a quantidade correspondente da outra fica multiplicada ou dividida pelo mesmo número. No primeiro caso, a proporcionalidade se chama direta e, no segundo, inversa; as grandezas se dizem diretamente ou inversamente proporcionais. (TRAJANO 1960, apud, LIMA 1997 p. 92).

Lima (1997 p. 93), salienta que um modelo tradicional "equivale a dizer que a grandeza y é diretamente proporcional à grandeza $x$ quando existe um número $a$ (chamado a constante de proporcionalidade) tal que $y=a x$ para todo valor de $\mathrm{x}$ ".

Garcia (2005, p. 195), em seus estudos, buscou situar a proporcionalidade no domínio da Álgebra, apresentando uma explicação para a organização clássica de proporcionalidade, enquanto igualdade entre grandezas. Observamos que os temas "redução a unidade" e "regra de três" citados, fazem parte do estudo setor "quarta proporcional" e, que o tema "equação do $1^{\circ}$ grau" pertencente ao setor "equação" fazem parte do domínio da Álgebra. O autor, ressalta também, que os temas "redução a unidade" e "regra de três" são técnicas "clássicas", para resolução de tarefas com proporcionalidade e, expresso por uma equação.

A primeira organização matemática (primeiro nível de algebrização) surge da modernização da linguagem usada para descrever a relação entre grandezas, dando origem a uma evolução de técnicas "clássicas" (regra de três e redução à unidade) e tecnologia da organização clássica. A relação de proporcionalidade não é mais caracterizada por proporções (como razões homogêneas iguais) a serem expressas por meio de equações: "duas grandezas $\mathrm{X}$ e Y são diretamente proporcionais ${ }^{7}$ quando uma correspondência entre elas pode ser estabelecida de tal maneira que, entre qualquer quantidade $\mathrm{x}$ de $\mathrm{X}$ e seu correspondente $\mathrm{y}$ de $\mathrm{Y}$ se cumpra a relação $\mathrm{y}=\mathrm{k}$. x "(Bolea, Bosch e Gascón, 2001b, p 276). Analogamente, a proporcionalidade inversa $^{8}$ simples será expressa pela equação $\mathrm{x} \cdot \mathrm{y}=\mathrm{k}, \mathrm{ou}, \mathrm{y}=\mathrm{k} / \mathrm{x}=\mathrm{k} \cdot \mathrm{x}^{-1}$.

\footnotetext{
${ }^{7}$ Grifo nosso.

${ }^{8}$ Grifo nosso.

${ }^{9}$ La primera organización matemática (primer nivel de algebrización) surge de la modernización del lenguaje usado para describir la relación entre magnitudes, dando lugar a una evolución de las técnicas "clásicas" (regla de tres y reducción a la unidad) y de la tecnología de la organización clásica. La relación de proporcionalidad deja de estar caracterizada mediante proporciones (como igualdad de razones homogéneas) para expresarse mediante ecuaciones: "dos magnitudes $\mathrm{X}$ e $\mathrm{Y}$ son directamente proporcionales cuando se puede establecer una correspondencia entre ellas de tal forma que, entre una cantidad cualquiera $\mathrm{x}$ de $\mathrm{X}$ y su correspondiente y de $\mathrm{Y}$ se
} 


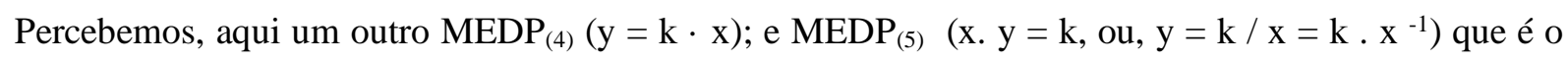
modelo identificado nesse recorte do texto de Garcia (2005). Onde, a primeira equação, corresponde as grandezas proporcionais diretas - função linear e a segunda, indica as grandezas proporcionais inversas - função exponencial.

Segundo, Lima (2010), o modelo matemático de proporcionalidade, “onde as grandezas são substituídas por números reais que são suas medidas". Considerando apenas as medidas positivas de grandezas, o "modelo matemático da proporcionalidade leva em consideração apenas números reais positivos". O nosso próximo $\operatorname{MEDP}_{(6)}$, remete ao Teorema Fundamental da Proporcionalidade que de acordo com o autor

Uma proporcionalidade (numérica) é uma função $f: \mathrm{R}+\rightarrow \mathrm{R}+$ com as seguintes propriedades:

1) $f$ é uma função crescente, isto é, $\mathrm{x}<\mathrm{x}^{\prime} \Rightarrow \mathrm{f}(\mathrm{x})<\mathrm{f}\left(\mathrm{x}^{\prime}\right)$ para quaisquer $\mathrm{x}, \mathrm{x}^{\prime}$ $\in \mathrm{R}+$.

2) Para todo $x \in R+$ e todo $n \in N$ tem-se $f(n x)=n$. $f(x)$.

Continuando, Lima (2010), modeliza função linear assegurando que

Uma função $f: \mathrm{R} \rightarrow \mathrm{R}$ definida por $f(x)=a x$, onde $a \in \mathrm{R}$ é uma constante chamada, chama-se função linear. Quando a > 0, a função linear $f(x)$ transforma um número real positivo $x$ no número positivo ax, e logo define, por restrição, uma proporcionalidade $f: \mathrm{R}+\rightarrow \mathrm{R}+$. Acabamos de ver que, reciprocamente, toda proporcionalidade é a restrição de uma função linear a $\mathrm{R}+$. A coeficiente $a$ chama-se o fator de proporcionalidade. LIMA (2010, p. 06-07).

Lima (2010, p. 02), destaca que para que exista uma proporcionalidade se faz necessário que se atenda a duas condições se $x \rightarrow y$ e $x^{\prime} \rightarrow y^{\prime}$, então, $x<x^{\prime}$ implica $y<y^{\prime}$; e, se $x \rightarrow y$ então $n x \rightarrow n y$, para todo $n \in \mathrm{N}$. Que estamos aqui tomando como um outro MEDP ${ }_{(7)}$.

1) Quanto maior for $x$, maior será $y$. Em termos matemáticos: se $x \rightarrow y$ e $x^{\prime} \rightarrow y^{\text {' }}$ então $x<x^{\prime}$ implica $y<y^{\prime}$.

2) Se dobrarmos, triplicarmos etc. o valor de $\mathrm{x}$, o valor correspondente de y será dobrado, triplicado etc. na linguagem matemática: se $x \rightarrow y$ então $n x \rightarrow$ ny para todo $n \in \mathrm{N}$.

Nas condições acima, a correspondência $x \rightarrow y$ chama-se uma proporcionalidade.

Bem entendido que, dependendo do problema matemático a ser solucionado, essa definição torna-se ineficiente como em contexto monetário, como exemplifica o autor.

cumpla la relación y = k·x" (Bolea, Bosch y Gascón, 2001b, p. 276). De manera análoga, la proporcionalidad simple inversa se expresará mediante la ecuación $\mathrm{x} \times \mathrm{y}=\mathrm{k}$, o bien, $\mathrm{y}=\mathrm{k} / \mathrm{x}=\mathrm{k} \times \mathrm{x}^{-1}$. 
Se uma quantia fixa gera, após um mês de investimento, um retorno y, não é verdade que após $n$ meses essa quantia gere o retorno $n$. y, mesmo que a taxa de juros permaneça constante. Pois ao final de cada mês é como se tivesse sido aplicada novamente uma quantia maior, igual a existente no mês anterior mais os juros correspondentes. Assim o retorno (num período fixo) é proporcional ao capital inicial, mas não é proporcional ao tempo de investimento. (LIMA, 2010).

Percebemos que, no $\operatorname{MEDP}_{(7)}$, em Lima (2010, p. 02), o atributo que se interpreta que "quanto maior for x maior será y" não garante a proporcionalidade entre x e y, como pode ser observado no exemplo dado em contexto monetário.

Entretanto, dois outros temas muito recorrentes, na matemática, do ensino fundamental e, que vez por outra se confundem no dia a dia ao saber proporcionalidade, são a proporção e a regra de três. Em vista disto pensamos prudente elucidar em nosso texto, neste momento. Recorremos a Lima (2010, p.07), quando aponta que

Se $f: \mathrm{R}+\rightarrow \mathrm{R}+$ é uma proporcionalidade, então, para quaisquer $x_{1}, x_{2} \operatorname{com} f\left(x_{1}\right)=y_{1}$, $f\left(x_{2}\right)=y_{2}$, tem-se $y_{1} / x_{1}=y_{2} / x_{2}$. Com efeito, ambos esses cocientes são iguais ao fator de proporcionalidade $a$. A igualdade $y_{1} / x_{1}=y_{2} / x_{2}$ chama-se proporçã ${ }^{10}$.

Chama-se de regra de três ${ }^{11}$ ao problema que consiste em, conhecendo três dos números $x_{1}, x_{2}, y_{1}, y_{2}$, determinar o quarto.

Identificamos também, como modelos dominantes relacionado ao saber proporcionalidade, os MEDP (8), “a igualdade $y_{1} / x_{1}=y_{2} / x_{2}$ chama-se de proporção", Lima (2010), e MEDP (9) regra de três, "ao problema que consiste em, conhecendo três dos números $x_{1}, x_{2}, y_{1}, y_{2}$, determinar o quarto”, Lima (2010).

Ainda, sobre proporção que tem principalmente, nicho ecológico nas grandezas, encontramos em Bellemain (2002, p. 76), uma citação de Heart (1956), do Livro V, dos Elementos de Euclides. "(Quatro) grandezas são ditas terem mesma razão, a primeira para a segunda e a terceira para a quarta, se os múltiplos da primeira e da terceira são correspondentes maiores, iguais ou menores do que os mesmos múltiplos da segunda e da quarta." Para essa citação, Bellemain (2002, p. 76), apresenta uma "tradução" em linguagem matemática, que a tomamos como MEDP (10), para proporção, explicitamente, o domínio das Grandezas.

Em notação atual: A igualdade $\frac{A}{B}=\frac{C}{D}$, é equivalente ao conjunto das seguintes implicações lógicas:

1. Se para $\mathrm{m}, \mathrm{n}$ inteiros, $\mathrm{mA}>\mathrm{nB}$, então, $\mathrm{mC} . \mathrm{nD}$.

2. Se para $m, n$ inteiros, $m A=n B$, então, $m C=n D$.

3. Se para $\mathrm{m}, \mathrm{n}$ inteiros, $\mathrm{mA}<\mathrm{nB}$, então, $\mathrm{mC}<\mathrm{nD}$.

Mas, proporcionalidade também tem nicho ecológico da Geometria, no habitando o setor semelhança. Encontramos uma referência, Lima (1991, p. 31), apresentando que "a noção de

${ }^{10}$ Grifo nosso.

${ }^{11}$ Idem. 
semelhança corresponde à ideia natural de "mudança de escala", isto é, ampliação ou redução de uma figura alterando seu tamanho sem modificar suas proporções”. Que tomamos como $\operatorname{MEDP}_{(11)}$. Mas, autor, alerta que toda figura é semelhante a si própria e remete a função identidade, $\sigma: F \rightarrow F^{\prime}$, que é uma semelhança de razão 1. Tomamos a função identidade como MEDP (12). Lima (1991, p. 33), apresenta a seguinte modelo para semelhança.

\footnotetext{
Sejam $F$ e $F$ ' figuras, do plano ou d o espaço, e $r$ um número real positivo. Diz-se que $F$ e $F$ ' são semelhantes, com razão de semelhança $r$, quando existe uma correspondência biunívoca $\sigma: F \rightarrow F^{\prime}$, entre os pontos de $F$ e os pontos de $F^{\prime}$, com a seguinte propriedade:

Se $X, Y$ são pontos quaisquer de $F$ e $X^{\prime}=\sigma(\mathrm{X}), Y^{\prime}=\sigma(Y)$ são seus correspondentes e F' então $\overline{x^{\prime} y^{\prime}}=r \cdot \overline{X Y}$.

A correspondência biunívoca $\sigma: F \rightarrow F^{\prime}$, com esta propriedade de multiplicar as distancias pelo fator constante $\mathbf{r}^{12}$, chama-se uma semelhança de razão $r$, entre $F \mathrm{e}$ $F^{\prime}$. Se $X^{\prime}=\sigma(X)$, diz-se que os pontos $X, Y$ são homólogos.
}

Os modelos epistemológicos dominantes para proporcionalidade identificados: MEDP (1) Ávila (1986), MEDP $_{(2)}$ Post, Behr e Lehs (1995), MEDP $_{(3)}$ Lima (1997), MEDP $(4)$ Garcia (2005) e MEDP (6) Lima (2010), adotarem a ideia de fator de proporcionalidade, representado ora pela letra " $k$ ", ora pela letra “ $a$ ", em ambos os casos, entendemos esses modelos fazem uso de expressões diferentes, porém, dando mesma conotação para a representação algébrica da proporcionalidade, enquanto função linear.

É interessante observar que nos textos dos livros de Lima (2010 e 1997) o "Teorema Fundamental da Proporcionalidade" é descrito pelo modelo da função linear, "função $f: \mathrm{R} \rightarrow$ $\mathrm{R}$ definida por $f(x)=a x$, onde $a \in \mathrm{R}$ é uma constante".

Em virtude dos modelos identificados, podemos concluir que a proporcionalidade, vem principalmente, dos domínios da álgebra, das grandezas e da geometria para se tornar objeto de estudo no ensino fundamental. Concluímos, também que, neste nível de ensino, a razão de ser da proporcionalidade se dá a partir dos MEDP identificados, onde proporcionalidade habita principalmente os setores função, grandezas proporcionalmente direta ou inversa e semelhança.

\section{Ecologia didática do saber proporcionalidade nos artigos a acadêmicos}

Nosso olhar para análise dos artigos acadêmicos buscou na TAD, elementos da ecologia didática, para responder também, as perguntas: como se dá a ecologia deste saber na literatura acadêmica? Onde habita este saber? Quais nichos possui?

${ }^{12}$ Grifo nosso, entendemos fator constante $r$, como fator de proporcionalidade $\mathrm{r}$. 
Foi possível observar que a proporcionalidade possui vida no ecossistema Matemática, que ela habita nas cinco subáreas: Álgebra, Estatística e probabilidade, Geometria, Grandezas e medidas e Números. No entanto, neste recorte da pesquisa, abordaremos apenas três, dos cinco habitats - Álgebra, Geometria e Número.

A matemática, em nosso estudo, atua como ecossistema onde o saber proporcionalidade habita e desempenha suas funções e interrelações. Em cada habitat, o saber possui nichos que se integram entre os diferentes habitats mantendo sua condição de vida no ecossistema.

Observamos nos artigos que a proporcionalidade habita a Álgebra, quando a ecologia das tarefas a função linear atua como principal nicho ecológicos. Como função, tem-se na proporção ou, no fator de proporcionalidade sua essência. (BORTOLOTI e BARBOSA, 2018, p. 289).

Quando habita a Geometria, a proporcionalidade tem na semelhança de figuras seu principal nicho, na ecologia de tarefas, envolvendo principalmente escala, ampliação/redução de figuras, teorema de Tales, e homotetia. A noção de semelhança corresponde a ideia natural de mudança de escala (LIMA, 1991, p. 31 apud MELO, 2004, p.44).

Porém, nossas leituras apontaram Número, como o habitat com a maior quantidade de nichos, para este saber. Foram encontrados, tanto nos enunciados dos tipos de tarefas quanto nas técnicas desenvolvidas, grande variedade de conexões, com dois principais nichos: Razão e Proporção. A Razão é um dos nichos com teias de interrelações bem ampla, ela se articula principalmente aos saberes: divisão proporcional, escala, porcentagem, quotização proporcional e taxa. Ao realizar uma tarefa envolvendo o nicho, Razão, as quantidades podem aumentar ou diminuir, mas um valor, ou seja, a razão sob a qual as grandezas variarão permanece para que a proporcionalidade seja mantida.

\section{Considerações finais}

Pelo fato de tratar-se de uma pesquisa em andamento ainda há muito caminho a ser percorrido no estudo da ecologia do saber proporcionalidade. No entanto, os resultados preliminares apontaram que as razões de ser da proporcionalidade se dá pelo fato deste saber transitar em diferentes esferas cognitiva, matemática e didática no ensino e da aprendizagem.

O estudo também permitiu a construção de dados relevantes acerca do saber de proporcionalidade a partir da análise da sua ecologia, nos artigos acadêmicos. O saber em questão, tem na Matemática da Educação Básica um ecossistema, no qual vive e desempenha 
seu papel de articulador nos diferentes habitats que faz parte. Habitando principalmente as subáreas da matemática Álgebra, Geometria e Números, tem como nicho ecológico, saberes relacionados a: razão, proporção, semelhança e função linear.

Esse recorte da pesquisa confirmou a importância do estudo de proporcionalidade, nos apontou caminhos, mas, ainda há muito a ser trilhado. Pretendemos continuar o estudo percorrendo outros textos acadêmicos - dissertações e teses, bem como, o livro didático e o referencial curricular.

\section{Referências}

BEN-CHAIM, D., ILANY, B. e KERET, Y. Atividades Investigativas Autênticas" para o Ensino de Razão e Proporção na Formação de Professores de Matemática para os Níveis Elementar e Médio. Bolema, Rio Claro (SP), Ano 21, no 31, 2008, p. 125 a 159.

BONGIOVANNI. V. As duas maiores contribuições de Eudoxo de Cnido "a teoria das proporções e o método da exaustão". Revista Ibero-americana de Educação Matemática. 2005. $\mathrm{N}^{\mathrm{o}} 02$, p. $91-110$.

BORTOLOTI, R. D. M; BARBOSA, J. C. Matemática para o ensino do conceito de proporcionalidade a partir de um estudo do conceito. Educ. Matem. Pesq., São Paulo, v.20, n.1, pp. 269-293, 2018

CHAACHOUA, H. Le role de l'analyse des manuels dans la Theorie Antopologique du Didactique. 2014. <hal-01519339>. Arquivo digital.

CHEVALLARD, Y. Les processus de transposition didactique et leur theorisation. 1994. Arquivo eletrônico.

Questions vives, savoirs moribonds: le problème curriculaire

aujourd'hui: 1997. Arquivo eletrônico.

2010, 139-146.

La didactique, dites-vous? Education\& didactique, 4.1, outubro de

COMIN, E. Proportionnalité et fonction linéaire. Caracteres, causes et effets didactiques des évolutions et didactitiques des évollutions et des réforms dans la scolarité obligatoire. Thése presentée à l'Universite Bordeaux I. 2000.

COSTAS, M. DOS S. \& AlleVAto. N. S, G. O Conceito de Proporcionalidade através da Resolução de Problemas de Geometria: perspectivas didáticas de (futuros) professores de Matemática em formação inicial. www2.rc.unesp.br/gterp/sites/default/ files/artigos/ trab_completo_manoel.pdf. Acesso em 03.01.17

FONSECA, J. C. P. Variação-proporção: um novo formato para apresentação de dados. Revista Administração Empresarial, Out 1974, vol.14, no.5, p.74-79. ISSN 00347590. 2018. Arquivo digital. 
IMENES, L. M. A Proporcionalidade sob o Ponto de Vista da Transposição Didática com Luiz Márcio Imenes pelas pesquisadoras Changkuo Rodrigues e Dayane Cristina Rocha Tinoco. Cadernos da Educação Básica, vol. 1, n. 3, janeiro 2017.

MELO, M. S. L. de. Um estudo sobre o ensino e aprendizagem do conceito de escala, no quarto ciclo do Ensino Fundamental. Dissertação (Mestrado em Educação) - Programa de Pós-Graduação em Educação. Centro de Educação. Universidade Federal de Pernambuco. Recife, 2004.

POST, R. T; BEHR, J. M. E; LESH. R. A Proporcionalidade e o desenvolvimento de noções pré-álgebra. São Paulo: Atual, 1995.

ORDOÑEZ. E. A. S. Razones, proporciones y proporcionalidade en una situacón de reparto: una mirada desde la Teoría Antropológica de lo Didáctico. Revista Latinoamericana de Investigación en Matemática Educativa (2013) 16 (1): 65-97.

SANTOS, M; MENEZES, M. B. A Teoria Antropológica do Didático: uma Releitura Sobre a Teoria. Perspectivas da Educação Matemática - UFMS - v. 8, número temático 2015.

SPINILlO, A. G. Papel de Intervenções Específicas na Compreensão da Criança sobre Proporção. Psicologia: Reflexão e Crítica, 2002, 15(3), pp. 475-487. 